\title{
Nanomechanical Characterization of the Kondo Charge Dynamics in a Carbon Nanotube
}

\author{
K. J. G. Götz, D. R. Schmid, F. J. Schupp, P. L. Stiller, Ch. Strunk, and A. K. Hüttel \\ Institute for Experimental and Applied Physics, University of Regensburg, Universitätsstraße 31, 93053 Regensburg, Germany
}

(Received 1 February 2018; revised manuscript received 23 April 2018; published 14 June 2018)

\begin{abstract}
Using the transversal vibration resonance of a suspended carbon nanotube as a charge detector for its embedded quantum dot, we investigate the case of strong Kondo correlations between a quantum dot and its leads. We demonstrate that even when large Kondo conductance is carried at odd electron number, the charging behavior remains similar between odd and even quantum dot occupations. While the Kondo conductance is caused by higher order processes, a sequential tunneling only model can describe the time-averaged charge. The gate potentials of the maximum current and fastest charge increase display a characteristic relative shift, which is suppressed at increased temperature. These observations agree very well with models for Kondo-correlated quantum dots.
\end{abstract}

DOI: 10.1103/PhysRevLett.120.246802

The Kondo effect [1] is a striking manifestation of electronic correlations. In semiconductor quantum dots as Coulomb blockade systems [2], in its most prevalent type it expresses itself as a distinct zero-bias maximum of differential conductance at odd electronic occupation [3-5]. In spite of this strong impact on electronic charge transport, the degeneracy central to its formation is then given by the spin states of an unpaired electron: for the SU(2) spin Kondo effect, below a characteristic temperature $T_{K}$, exchange coupling between a localized electron and conduction band charges leads to the formation of the Kondo resonance at the Fermi level. A question that arises immediately is how the strongly enhanced Kondo conductance within Coulomb blockade relates to the precise charge trapped within the quantum dot and its evolution as a function of applied gate voltage [6-8].

Suspended carbon nanotube quantum dots provide extraordinarily clean and controllable mesoscopic model systems $[9,10]$, where transport spectra from single and few electron physics [11-14] all the way to open systems and electronic Fabry-Perot interferometry $[15,16]$ can be analyzed. Also regarding Kondo phenomena, a wide range of experimental work on carbon nanotubes exists [17-21], making use of the well-characterized electronic structure. Then again, as nanoelectromechanical systems, carbon nanotubes have shown at cryogenic temperatures exceedingly high mechanical quality factors [22-24] and strong interaction between single electron tunneling and vibrational motion [25-28]. The detection of the transversal vibration frequency of a carbon nanotube provides a powerful means to measure the charge on its embedded quantum dot $[25,28]$.

In this Letter, we investigate the parameter region of strong Kondo correlations between a suspended nanotube quantum dot and its metallic leads [3-5,17-21,29]. We measure the gate voltage dependence of the time-averaged charge $e\langle N\rangle\left(V_{\mathrm{g}}\right)$ on the quantum dot. The observed typical asymmetry in conductance between odd and even occupation states, indicating SU(2) Kondo behavior, is clearly absent in the gate-dependent trapped charge. This shows that the current is carried by higher-order processes leading to only virtual occupation on the quantum dot, while the time-averaged charge remains determined by the first-order processes of sequential tunneling. In addition, we observe a distinct gate voltage offset between charging of the quantum dot and the current maximum, which is suppressed at increasing temperature. Our results agree very well with theoretical studies of Kondo-correlated quantum dots $[6,30]$.

Device characterization.-Figure 1(a) displays a sketch of our device structure; a table of fabrication parameters can be found in the Supplemental Material [31]. On a highly $p^{++}$doped $\mathrm{Si}$ substrate with thermally grown $\mathrm{SiO}_{2}$ on top, electrode patterns were defined via electron beam lithography and metal evaporation. The metal layer directly serves as an etch mask for subsequent anisotropic dry etching of the oxide, generating deep trenches between the electrodes. As last steps, a growth catalyst was locally deposited, and carbon nanotubes were grown via chemical vapor deposition [32].

Electronic transport measurements were performed in a dilution refrigerator at $T_{\mathrm{MC}} \leq 25 \mathrm{mK}$. The measurement setup combines a dc current measurement as required for Coulomb blockade transport spectroscopy [35] with radio-frequency irradiation using an antenna several millimeters from the device $[22,23,25]$. As can be seen from the differential conductance in linear response in Figs. 1(b) and 1(c), in both devices close to a small band gap Coulomb blockade and sequential tunneling dominates. For larger positive gate voltages $V_{g}$, the transparency of the tunneling barriers increases. This leads to a crossover towards regular Kondo enhancement of the conductance 

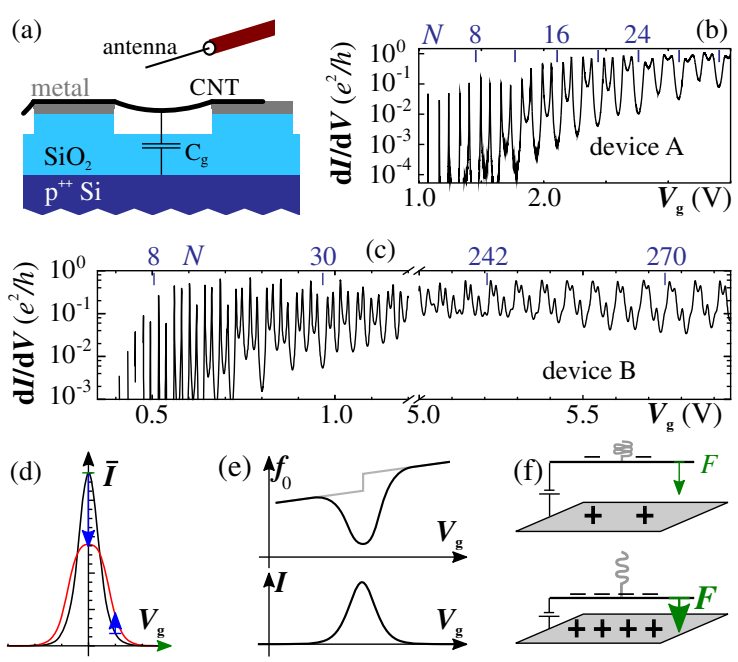

FIG. 1. (a) Sketch of the device geometry (not to scale). See the Supplemental Material [31] for a table of the device properties. (b),(c) Low-bias differential conductance $d I / d V_{\text {sd }}$ of carbon nanotube devices $A$ and $B$ as a function of applied back gate voltage $V_{g} . N$ indicates the number of trapped electrons. The transition from strong Coulomb blockade (left edge) to strongly Kondo enhanced transport is visible in both cases. (d) At resonant driving, a nanotube vibrates strongly, leading to a fast oscillation of $C_{g}$ and, averaged over the vibration, a broadening of Coulomb oscillations. (e) Typical gate voltage evolution of the transversal vibration resonance frequency and the current in the Coulomb blockade regime, see [25] and the text. (f) Principle of electrostatic vibration softening: when a vibrating capacitor at constant voltage adapts its charge to the momentary position, an electrostatic force opposite to the mechanical restoring force occurs. This results in an effective smaller spring constant and resonance frequency.

$[4,17]$; the clear twofold pattern in Fig. 1(b) indicates approximate $\mathrm{SU}(2)$ Kondo behavior [36].

Mechanical resonance detection.-With a radiofrequency signal applied at the mechanical resonance $f_{0}$ of its transversal vibration mode, the nanotube is driven into motion, leading to a change in detected dc current [22,23,25,28]: the capacitance between back gate and nanotube $C_{g}$ is modulated with the deflection, broadening the Coulomb oscillations in a slow dc measurement, as shown in Fig. 1(d). This enables us to detect $f_{0}$ and its dependence on the back gate voltage $V_{g}$ in the dc current.

Figure 1(e) sketches a typical evolution of the resonance frequency with increasing positive gate voltage in the strong Coulomb blockade regime [25]. The continuous increase of the gate charge and the discrete increase of the quantum dot charge both contribute via mechanical tension to $f_{0}$, as continuous increase and step function, respectively. Further, when the electronic tunnel rates are large compared to $f_{0}$, near charge degeneracy points the charge on the quantum dot can adapt (by a fraction of an elementary charge) to the momentary position within a vibration cycle. The vibration mode is electrostatically softened [25,38], cf. Fig. 1(f), proportional to $\partial\langle N\rangle / \partial V_{g}$. Thus, resonance frequency minima indicate the increase of the quantum dot charge $e\langle N\rangle$ at the charge degeneracy points [25,38] and $\langle N\rangle\left(V_{g}\right)$ can be calculated from the frequency evolution $f_{0}\left(V_{g}\right)$.

Figure 2(a) shows a measurement of the vibrationinduced signal in the Kondo regime. For different gate voltages $V_{g}$, the time-averaged dc current $I\left(V_{g}, f\right)$ is recorded while sweeping the driving signal frequency $f$. In Fig. 2(a) [and Fig. 2(c), which displays a trace cut from Fig. 2(a)], the mean value $\bar{I}\left(V_{g}\right)$ of each frequency sweep has been subtracted for better contrast. The vibration resonance becomes clearly visible as a diagonal feature. To evaluate its detailed evolution, we extract $f_{0}\left(V_{g}\right)$ and plot it in Fig. 2(d) as $\tilde{\omega}_{0}\left(V_{g}\right)=2 \pi f_{0}\left(V_{g}\right)-\left(a+b V_{g}\right)$, i.e., with a linear background subtracted [39]. Every singleelectron addition into the dot exhibits a distinct dip. While the off-resonant dc current $I\left(V_{g}\right)$, Fig. 2(b), clearly shows Kondo zero bias conductance anomalies at an odd quantum dot charge [4], this odd-even electron number asymmetry is barely visible in the evolution of the resonance frequency [40].

Evolution with $V_{g}$.- In Fig. 3(a), we show a detail of the resonance frequency evolution from Fig. 2(d), accompanied by the current $I\left(V_{g}\right)$ in Fig. 3(b). To model it, we reduce the quantum dot to two nondegenerate Lorentzbroadened levels, separated by a capacitive addition energy

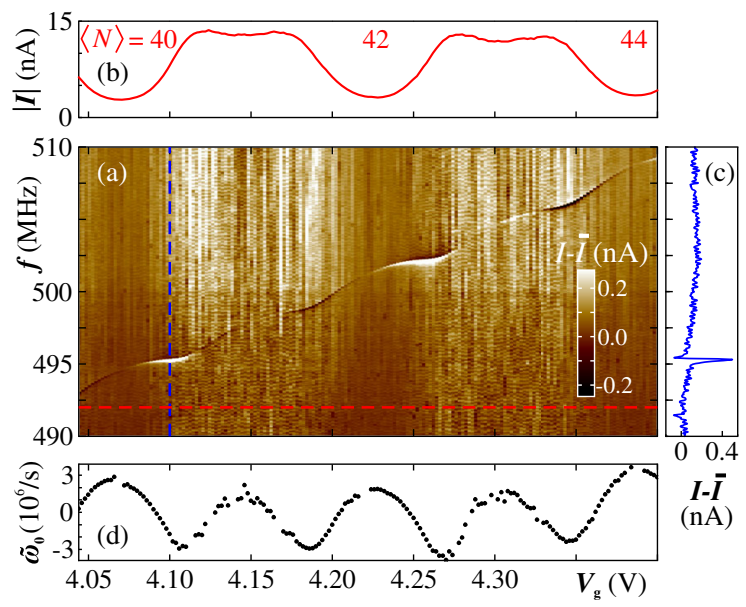

FIG. 2. (a) Current through the quantum dot, as a function of gate voltage $V_{g}$ and $\mathrm{rf}$ driving frequency $f$, with the mean current $\bar{I}\left(V_{g}\right)$ of each frequency trace subtracted; nominal rf generator power $-25 \mathrm{dBm}$, bias voltage $V_{\text {sd }}=-0.1 \mathrm{mV}$. (b) $\left|I\left(V_{g}\right)\right|$ at offresonant driving frequency $f=492 \mathrm{MHz}$. Kondo enhanced conductance occurs at odd electron numbers. (c) Example trace $I\left(V_{g}, f\right)-\bar{I}\left(V_{g}\right)$ from (a) at $V_{g}=4.1 \mathrm{~V}$. The effect of the mechanical resonance on the time-averaged dc current is clearly visible. (d) Extracted resonance frequency shift $\tilde{\omega}_{0}\left(V_{g}\right)=$ $2 \pi f_{0}\left(V_{g}\right)-\left(a+b V_{g}\right)$ with respect to a linear background; see the Supplemental Material [31] for the detailed fit parameters. Device $A$. 


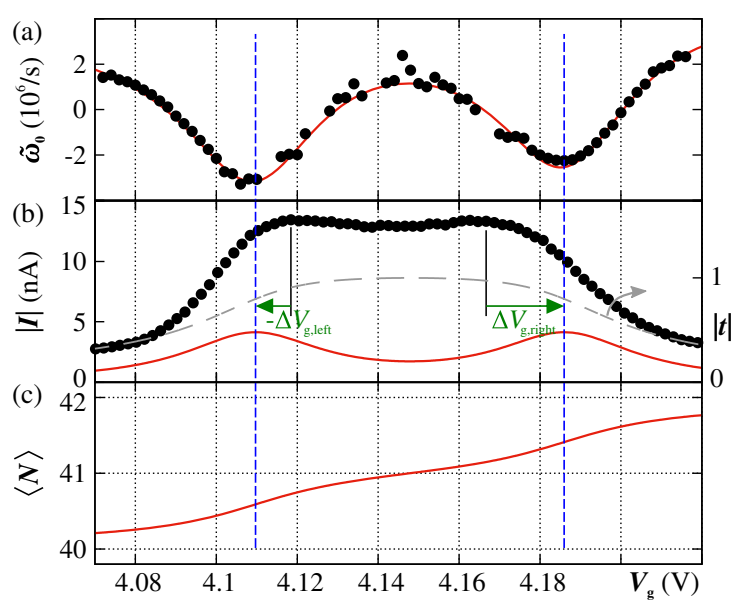

FIG. 3. Analysis of the Kondo regime around $\langle N\rangle=41$; $V_{\text {sd }}=-0.1 \mathrm{mV}$. (a) Data points: resonance frequency shift $\tilde{\omega}_{0}\left(V_{g}\right)$, cf. Fig. 2(d). Solid line: curve fit assuming subsequent occupation of two nondegenerate levels, see the text. (b) Data points: simultaneously measured off-resonant current $|I|\left(V_{g}\right)$. Solid red line: sequential tunneling current according to the fit model from (a). Dashed gray line: $T=0, V_{\text {sd }}=0$ Fermi liquid model transmission $|t|$ derived from $\langle N\rangle\left(V_{g}\right)$ via Friedel's sum rule, see the text; right axis. (c) Time-averaged quantum dot occupation $\langle N\rangle\left(V_{g}\right)$ derived from the fit in (a).

$U>\Gamma$, without taking any higher-order tunneling effects into account. We only consider the case of linear response, i.e., $e V_{\mathrm{sd}} \ll \hbar \Gamma$; in addition, for the large transparency of the contact barriers present at $N \approx 41$ and for electron temperatures of roughly $T \lesssim 50 \mathrm{mK}$, we neglect the thermal broadening of the Fermi distribution in the contacts. Then, $\langle N\rangle\left(V_{g}\right)$ is only smeared out by the lifetime broadening $\Gamma$ of the quantum dot states. The tunnel barrier transmittances between dot and leads are assumed to be energy independent and equal; the tunnel rates $\Gamma_{1 / 2}^{ \pm}$onto and off the quantum dot levels are obtained by integrating over the density of states on the dot.

We use this model to fit the functional dependence of the resonance frequency to the data in Fig. 3. Following $[25,28]$, the decrease of the resonance frequency at finite single electron tunneling [cf. Fig. 1(e)] is given by

$$
\Delta \omega_{0}=\frac{V_{g}\left(V_{g}-V_{\mathrm{CNT}}\right)}{2 m \omega_{0} C_{\Sigma}}\left(\frac{d C_{g}}{d z}\right)^{2}\left(1-\frac{e}{C_{g}} \frac{\partial\langle N\rangle}{\partial V_{g}}\right),
$$

with $V_{\mathrm{CNT}}=\left(C_{g} V_{g}-e\langle N\rangle\right) / C_{\Sigma}$ as the voltage on the CNT, $m$ the nanotube mass, and $\omega_{0}=2 \pi f_{0}$. The gate and total capacitances $C_{g}$ and $C_{\Sigma}$ are extracted from Coulomb blockade measurements. Since we do not know the precise position of our CNT, we treat the capacitive displacement sensitivity $d C_{g} / d z$, where $z$ is the deflection of the nanotube, as a free parameter. A detailed discussion of the fit procedure, a table of the device parameters entering the calculation, and the resulting fit parameters can be found in the Supplemental Material [31]. Note that the relevant gate dependent term in Eq. (1) is the quantum capacitance, i.e., the derivative of the charge occupation $\partial\langle N\rangle / \partial V_{g}$, also called compressibility in [8].

Our simplified model reproduces the functional dependence of the resonance frequency in Fig. 3(a) very well. The result can be used to derive the expected sequentialtunneling current from our model and the time-averaged charge evolution $\langle N\rangle\left(V_{g}\right)$ in the quantum dot, see the solid lines in Fig. 3. While Kondo processes absent in our model strongly contribute to electronic transport, they do not significantly influence the time-averaged occupation of the quantum dot and thereby the mechanical resonance. This is in excellent agreement with results by Sprinzak et al. [7], combining a quantum point contact as a charge detector $[41,42]$ with a gate-defined quantum dot, as well as recent data analyzing the charge compressibility of a quantum dot by means of a coupled coplanar waveguide cavity, see [8]. The suppression of quantum dot charging by Coulomb blockade is independent of the Kondo enhanced conductance via virtual occupation.

Gate potential of current and compressibility maxima.In a naive analogy, one would expect that in the Kondo case, as in the case of strong Coulomb blockade $[8,25,28]$, the increase of the time-averaged charge on the quantum dot takes place predominantly at the gate voltage of the current maxima. The data points of Fig. 3(b) show the current $I\left(V_{g}\right)$ at fixed bias, recorded simultaneously with the mechanical resonance frequency, Fig. 3(a). Comparing the extrema of the resonance frequency $\tilde{\omega}_{0}\left(V_{g}\right)$, Fig. 3(a), and the current $|I|\left(V_{g}\right)$, Fig. 3(b), distinct shifts $\Delta V_{g \text {, left }}$ and $\Delta V_{g, \text { right }}$ are observed, see the green arrows.

In the experimental literature, a temperature-induced shift of the current maximum due to Kondo correlations has already been reported in the first publications [3]. In the data of Sprinzak et al., [7], a systematic shift between current and quantum capacitance extrema similar to our observations is visible (though not discussed). This confirms that the phenomenon is intrinsic to the Kondo effect in a quantum dot, independent of the experimental realization. Early calculations by Wingreen and Meir, [30], using the noncrossing approximation in the Anderson model, have already predicted a temperature-dependent shift of the current maximum position (see Figs. 6 and 7(a) in [30]).

Temperature dependence.-Figure 4 illustrates the suppression of correlation effects at elevated temperature. In the region of the figure, we obtain Kondo temperatures in the range $1 \mathrm{~K} \lesssim T_{K} \lesssim 5 \mathrm{~K}$. While the large dot-lead coupling strongly distorts the stability diagram at base temperature [19,43], see Fig. 4(a), at $T \gtrsim 5 \mathrm{~K}$ in Fig. 4(b) regular, thermally broadened Coulomb blockade oscillations reemerge. Figures 4(c)-4(e) display both extracted mechanical resonance frequency and measured dc current for (c) $T=15 \mathrm{mK}$, (d) $T=0.7 \mathrm{~K}$, and (e) $T=5 \mathrm{~K}$. With increasing temperature, the mechanical resonance broadens [22], and the determination of the resonance frequency 

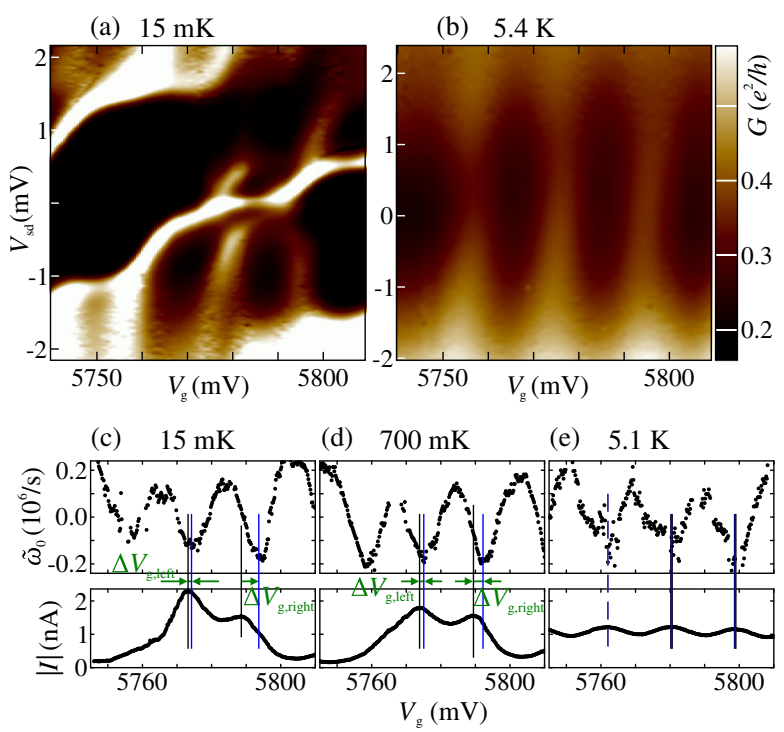

FIG. 4. (a),(b) Differential conductance of device $B$ at (a) base temperature $T=15 \mathrm{mK}$ and (b) $T=5.4 \mathrm{~K}$. While transport in the millidegrees Kelvin regime is dominated by higher order effects, above $T=5 \mathrm{~K}$ regular, strongly broadened Coulomb blockade oscillations emerge. (c)-(e) Combined plots of mechanical resonance shift $\tilde{\omega}_{0}\left(V_{g}\right)$ and dc current $|I|\left(V_{g}\right)$, for $V_{\text {sd }}=-0.1 \mathrm{mV}$ and (c) $T=15 \mathrm{mK}$, (d) $0.7 \mathrm{~K}$, (e) $5.1 \mathrm{~K}$.

becomes more challenging. At the same time, the current evolves from a complex, Kondo and level renormalization dominated behavior to broadened but regular and, in the plotted range, nearly bias-independent Coulomb blockade oscillations.

As expected, at higher temperature, charging and current maxima coincide better. This is quantified in Fig. 5, where the relative shifts in gate voltage $\Delta V_{g \text {,left }}$ and $\Delta V_{g \text {,right }}$ between resonance frequency minimum and current maximum are plotted for two exemplary Coulomb oscillations marked in Fig. 4. Starting from about $1.5 \mathrm{mV}$, respectively $5.2 \mathrm{mV}$, the peak shifts decrease with increasing temperature asymptotically towards zero. The straight lines in the figure, fits excluding the $T=15 \mathrm{mK}$ point due to likely saturation there, correspond to the typical logarithmic
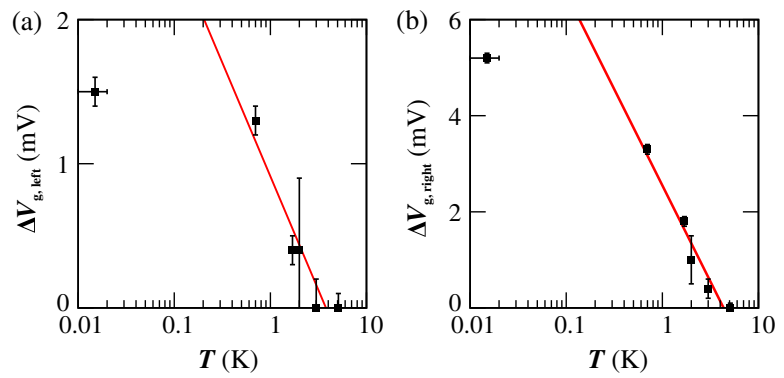

FIG. 5. Temperature dependence of (a) $\Delta V_{g, \text { left }}$ and (b) $\Delta V_{g, \text { right }}$, the shift between mechanical resonance frequency minimum and dc current maximum, for the oscillations marked in Fig. 4. The solid lines correspond to a logarithmic fit, with the $T=15 \mathrm{mK}$ point omitted [30]. scaling present in Kondo phenomena and predicted for the peak shift [30] and are consistent with the data.

Relation to the transmission phase.-In an early theoretical work on Kondo physics, Gerland et al. [6] discuss the electronic transmission phase of a Kondo quantum dot, a topic of intense attention over the previous decades. Friedel's sum rule $[44,45]$ intrinsically relates the transmission phase to the number of electronic states below the Fermi energy and thereby the time-averaged occupation. This means that we can directly compare the combined Figs. 3(c) and 3(d) of [6] (transmission magnitude and phase) with our data of Figs. 3(b) and 3(c) here (current and time-averaged occupation). Indeed, a highly similar functional dependence is visible; see the Supplemental Material [31] for a detailed comparison. With this background and based on Fermiliquid theory of the $S U(2)$ Kondo effect, the dashed gray line in Fig. 3(b) plots the transmission amplitude evolution $\left|t\left(V_{g}\right)\right|=\sin \left(\pi\langle N\rangle\left(V_{g}\right) / 2\right)$ of the quantum dot expected for $V_{\text {sd }}=T=0$. This clearly demonstrates the Kondo ridge as well as the distinct shift between a large transmission magnitude and maximum slope of the transmission phase. The deviations in current behavior $I\left(V_{g}\right)$ may be due to the finite temperature and bias and/or indicate an experimental situation more complex than the SU(2) Kondo effect.

Conclusion.-We use the mechanical resonance frequency of a suspended carbon nanotube to trace the average electronic occupation of a strongly Kondo-correlated quantum dot embedded in the nanotube. We show that sequential tunneling alone already provides a good model for the average charge $\langle N\rangle\left(V_{g}\right)$ and the mechanical resonance frequency $\omega_{0}\left(V_{g}\right)$. While dominant for electronic transport (conductance), the influence of Kondo correlations on the time-averaged charge and thereby the mechanical system is small in the chosen parameter regime. We observe a distinct shift in gate voltage of the current maxima, relative to the maxima of the charge compressibility $\partial\langle N\rangle / \partial V_{g}$, effectively distorting the Coulomb blockade regions. This shift decays with increasing temperature, a clear signature that it is caused by the Kondo correlations. Our results are in excellent agreement with theoretical modeling [6,30].

Future work, applying our highly versatile sensing method to higher harmonic modes of the vibration, may address the parameter region $f_{\text {mech }}>k_{B} T_{K}[46,47]$ or even the charge distribution along the carbon nanotube axis via a spatially modulated electron-vibration coupling [38]. Kondo phenomena in carbon nanotubes beyond the $\mathrm{SU}(2)$ spin Kondo effect provide further systems of obvious experimental and theoretical interest.

The authors acknowledge financial support by the Deutsche Forschungsgemeinschaft (Emmy Noether Grant No. Hu 1808/1, GRK 1570, and SFB 689) and by the Studienstiftung des deutschen Volkes. We thank J. von Delft, J. Kern, A. Donarini, M. Margańska, and M. Grifoni for insightful discussions. 
*andreas.huettel@ur.de

[1] J. Kondo, Resistance minimum in dilute magnetic alloys, Prog. Theor. Phys. 32, 37 (1964).

[2] C. W. J. Beenakker, Theory of Coulomb-blockade oscillations in the conductance of a quantum dot, Phys. Rev. B 44, 1646 (1991).

[3] D. Goldhaber-Gordon, J. Göres, M. A. Kastner, H. Shtrikman, D. Mahalu, and U. Meirav, From the Kondo Regime to the Mixed-Valence Regime in a Single-Electron Transistor, Phys. Rev. Lett. 81, 5225 (1998).

[4] D. Goldhaber-Gordon, H. Shtrikman, D. Mahalu, D. Abusch-Magder, U. Meirav, and M. A. Kastner, Kondo effect in a single-electron transistor, Nature (London) 391, 156 (1998).

[5] S. M. Cronenwett, T. H. Oosterkamp, and L. P. Kouwenhoven, A tunable Kondo effect in quantum dots, Science 281, 540 (1998).

[6] U. Gerland, J. von Delft, T. A. Costi, and Y. Oreg, Transmission Phase Shift of a Quantum Dot with Kondo Correlations, Phys. Rev. Lett. 84, 3710 (2000).

[7] D. Sprinzak, Y. Ji, M. Heiblum, D. Mahalu, and H. Shtrikman, Charge Distribution in a Kondo-correlated Quantum Dot, Phys. Rev. Lett. 88, 176805 (2002).

[8] M. M. Desjardins, J. J. Viennot, M.C. Dartiailh, L. E. Bruhat, M. R. Delbecq, M. Lee, M.-S. Choi, A. Cottet, and T. Kontos, Observation of the frozen charge of a Kondo resonance, Nature (London) 545, 71 (2017).

[9] J. Cao, Q. Wang, and H. Dai, Electron transport in very clean, as-grown suspended carbon nanotubes, Nat. Mater. 4, 745 (2005).

[10] E. A. Laird, F. Kuemmeth, G. A. Steele, K. Grove-Rasmussen, J. Nygård, K. Flensberg, and L. P. Kouwenhoven, Quantum transport in carbon nanotubes, Rev. Mod. Phys. 87, 703 (2015).

[11] V. V. Deshpande and M. Bockrath, The one-dimensional Wigner crystal in carbon nanotubes, Nat. Phys. 4, 314 (2008).

[12] F. Kuemmeth, S. Ilani, D. C. Ralph, and P. L. McEuen, Coupling of spin and orbital motion of electrons in carbon nanotubes, Nature (London) 452, 448 (2008).

[13] S. Pecker, F. Kuemmeth, A. Secchi, M. Rontani, D. C. Ralph, P. L. McEuen, and S. Ilani, Observation and spectroscopy of a two-electron Wigner molecule in an ultraclean carbon nanotube, Nat. Phys. 9, 576 (2013).

[14] M. Margańska, D. R. Schmid, A. Dirnaichner, P. L. Stiller, Ch. Strunk, M. Grifoni, and A. K. Hüttel, Shaping electron wave functions in a carbon nanotube with a parallel magnetic field, arXiv:1712.08545.

[15] W. Liang, M. Bockrath, D. Bozovic, J. H. Hafner, M. Tinkham, and H. Park, Fabry-Pérot interference in a nanotube electron waveguide, Nature (London) 411, 665 (2001).

[16] A. Dirnaichner, M. del Valle, K. J. G. Götz, F. J. Schupp, N. Paradiso, M. Grifoni, Ch. Strunk, and A. K. Hüttel, Secondary Electron Interference from Trigonal Warping in Clean Carbon Nanotubes, Phys. Rev. Lett. 117, 166804 (2016).

[17] J. Nygård, D. H. Cobden, and P. E. Lindelof, Kondo physics in carbon nanotubes, Nature (London) 408, 342 (2000).

[18] P. Jarillo-Herrero, J. Kong, H. S. J. van der Zant, C. Dekker, L. P. Kouwenhoven, and S. De Franceschi, Orbital Kondo effect in carbon nanotubes, Nature (London) 434, 484 (2005).

[19] A. Makarovski, J. Liu, and G. Finkelstein, Evolution of Transport Regimes in Carbon Nanotube Quantum Dots, Phys. Rev. Lett. 99, 066801 (2007).

[20] D. R. Schmid, S. Smirnov, M. Margańska, A. Dirnaichner, P. L. Stiller, M. Grifoni, A. K. Hüttel, and Ch. Strunk, Broken SU(4) symmetry in a Kondo-correlated carbon nanotube, Phys. Rev. B 91, 155435 (2015).

[21] M. Niklas, S. Smirnov, D. Mantelli, M. Margańska, N.-V. Nguyen, W. Wernsdorfer, J.-P. Cleuziou, and M. Grifoni, Blocking transport resonances via Kondo manybody entanglement in quantum dots, Nat. Commun. 7, 12442 (2016).

[22] A. K. Hüttel, G. A. Steele, B. Witkamp, M. Poot, L. P. Kouwenhoven, and H. S. J. van der Zant, Carbon nanotubes as ultra-high quality factor mechanical resonators, Nano Lett. 9, 2547 (2009).

[23] A. K. Hüttel, H. B. Meerwaldt, G. A. Steele, M. Poot, B. Witkamp, L. P. Kouwenhoven, and H. S. J. van der Zant, Single electron tunneling through high-Q single-wall carbon nanotube NEMS resonators, Phys. Stat. Solidi B 247, 2974 (2010).

[24] J. Moser, A. Eichler, J. Güttinger, M. I. Dykman, and A. Bachtold, Nanotube mechanical resonators with quality factors of up to 5 million, Nat. Nanotechnol. 9, 1007 (2014).

[25] G. A. Steele, A. K. Hüttel, B. Witkamp, M. Poot, H. B. Meerwaldt, L. P. Kouwenhoven, and H. S. J. van der Zant, Strong coupling between single-electron tunneling and nanomechanical motion, Science 325, 1103 (2009).

[26] B. Lassagne, Y. Tarakanov, J. Kinaret, D. Garcia-Sanchez, and A. Bachtold, Coupling mechanics to charge transport in carbon nanotube mechanical resonators, Science 325, 1107 (2009).

[27] D. R. Schmid, P. L. Stiller, Ch. Strunk, and A. K. Hüttel, Magnetic damping of a carbon nanotube nano-electromechanical resonator, New J. Phys. 14, 083024 (2012).

[28] H. B. Meerwaldt, G. Labadze, B. H. Schneider, A. Taspinar, Y. M. Blanter, H. S. J. van der Zant, and G. A. Steele, Probing the charge of a quantum dot with a nanomechanical resonator, Phys. Rev. B 86, 115454 (2012).

[29] M. N. Kiselev, K. A. Kikoin, L. Y. Gorelik, and R. I. Shekhter, Kondo force in shuttling devices: Dynamical probe for a Kondo cloud, Phys. Rev. Lett. 110, 066804 (2013).

[30] N. S. Wingreen and Y. Meir, Anderson model out of equilibrium: Noncrossing-approximation approach to transport through a quantum dot, Phys. Rev. B 49, 11040 (1994).

[31] See Supplemental Material at http://link.aps.org/ supplemental/10.1103/PhysRevLett.120.246802, which includes Refs. [6,14,16,25,28,32-34].

[32] J. Kong, H. T. Soh, A. M. Cassell, C. F. Quate, and H. Dai, Synthesis of individual single-walled carbon nanotubes on patterned silicon wafers, Nature (London) 395, 878 (1998).

[33] E. D. Minot, Y. Yaish, V. Sazonova, and P. L. McEuen, Determination of electron orbital magnetic moments in carbon nanotubes, Nature (London) 428, 536 (2004).

[34] S. Reinhardt, C. Butschkow, S. Geissler, A. Dirnaichner, F. Olbrich, C. Lane, D. Schröer, and A. K. Hüttel, Lab::Measurement - a portable and extensible framework 
for controlling lab equipment and conducting measurements, arXiv:1804.03321.

[35] L. P. Kouwenhoven, C. M. Marcus, P. L. McEuen, S. Tarucha, R. M. Westervelt, and N.S. Wingreen, Electron Transport in Quantum Dots, in Mesoscopic Electron Transport, edited by L. L. Sohn, L. P. Kouwenhoven, and G. Schön (Kluwer, Dordrecht, The Netherlands, 1997).

[36] The maximum conductance value lower than $2 e^{2} / h$ is likely due to asymmetric tunnel barrier transparencies [37]. A larger version of Figs. 1(b) and 1(c) can be found in the Supplemental Material [31] as Fig. S1.

[37] T. K. Ng and P. A. Lee, On-site Coulomb Repulsion and Resonant Tunneling, Phys. Rev. Lett. 61, 1768 (1988).

[38] A. Benyamini, A. Hamo, S. V. Kusminskiy, F. von Oppen, and S. Ilani, Real-space tailoring of the electron-phonon coupling in ultraclean nanotube mechanical resonators, Nat. Phys. 10, 151 (2014).

[39] We use the angular frequency notation to closely follow the published literature [28]. See the Supplemental Material at http://link.aps.org/supplemental/10.1103/PhysRevLett.120 .246802 for the detailed fit parameters.

[40] As derived in [22], the amplitude of the resonance peak scales with $\mathrm{d}^{2} I / \mathrm{d} V_{\mathrm{g}}^{2}$, leading to only a weak resonance signal on the Kondo ridge and a corresponding increased scatter of the extracted center frequencies there.

[41] M. Field, C. G. Smith, M. Pepper, D. A. Ritchie, J. E. F. Frost, G. A. C. Jones, and D. G. Hasko, Measurements of Coulomb Blockade with a Noninvasive Voltage Probe, Phys. Rev. Lett. 70, 1311 (1993).

[42] A. K. Hüttel, S. Ludwig, H. Lorenz, K. Eberl, and J. P. Kotthaus, Direct control of the tunnel splitting in a oneelectron double quantum dot, Phys. Rev. B 72, 081310(R) (2005).

[43] The observed complex transport spectrum of device $B$ in this parameter range goes beyond the SU(2) Kondo model.

[44] J. Friedel, On some electrical and magnetic properties of metallic solid solutions, Can. J. Phys. 34, 1190 (1956).

[45] D. C. Langreth, Friedel Sum rule for Anderson's model of localized impurity states, Phys. Rev. 150, 516 (1966).

[46] E. A. Laird, F. Pei, W. Tang, G. A. Steele, and L. P. Kouwenhoven, A high quality factor carbon nanotube mechanical resonator at $39 \mathrm{GHz}$, Nano Lett. 12, 193 (2012).

[47] R. Delagrange, J. Basset, H. Bouchiat, and R. Deblock, Emission noise and high frequency cut-off of the Kondo effect in a quantum dot, Phys. Rev. B 97, 041412(R) (2018). 